\title{
Electrostatic Damping on the Motion of a Charged Particle in a Plasma
}

\author{
Christopher K. W. TAM \\ Guggenheim Jet Propulsion Center, California Institute of Technology, Pasadena, California
}

(Received 2 August 1965; final manuscript received 26 November 1965)

\begin{abstract}
The electrohydrodynamic phenomena associated with the motion of a charged particle in a plasma are investigated. It is shown that the type of disturbance produced in the plasma depends significantly on the speed of the particle, i.e., whether it is subsonic, transonic, or supersonic. In the subsonic case, the electrohydrodynamic drag experienced by the particle is caused by an unsymmetric wake formed by the screening electrons. In the supersonic case, electron screening is confined to the Mach cone. Ahead of the Mach cone, the plasma behaves as if it were an incompressible fluid. The electrohydrodynamic drag in this case has a logarithmic nature.
\end{abstract}

\section{INTRODUCTION}

$\mathrm{T}$ 'HE problem of an ion moving through a plasma has been considered by various authors from different points of view. In particular, Kraus and Watson ${ }^{1}$ have approached this problem by regarding the plasma as two coexisting ion and electron fluids. Their consideration, however, is restricted to the case of supersonic particles, as they were interested in the motion of satellites in the ionosphere. Moreover, their investigation does not show explicitly the response of the plasma to the particle motion. In this paper, the full range of the particle velocity is considered, i.e., subsonic, transonic, as well as supersonic velocity. Here, the plasma is also described by the two fluids model. A collision term, $\nu\left(\mathbf{u}_{e}-\mathbf{u}_{i}\right)$, is incorporated in the fluid equations to account for ion-electron interaction in the plasma. It is shown that the inclusion of this damping phenomenon permits the linearized fluid equations to describe the entire range of particle velocity without a mathematical breakdown, which in the case of conventional gasdynamics occurs when the Mach number approaches unity.

The result of this paper is applicable to a charged object moving through a relatively dense plasma at a moderate temperature. At these temperatures and densities, it is known that long range interaction forces prevail and the plasma exhibits a collective fluid-like behavior. The size of the object is considered to be sufficiently small so that the interaction between the object and plasma is dominated by Coulomb forces. Alternatively, the result can also be regarded as the response of a relatively dense plasma to the motion of a test particle. The response can be considered as composed of two parts, namely, that of the ions and electrons, re-

${ }^{1}$ L. Kraus and K. M. Watson, Phys. Fluids 1, 480 (1958). spectively. The ions are massive so that their perturbed motion is small compared to that of the electrons (the velocity ratio being inversely proportional to their mass ratio). Here we are primarily concerned with the response of the electrons in a scale much greater than the size of the object. In this respect, the motion of the ions is relatively unimportant and can be neglected as a first approximation (the error is of the order of the mass ratio). Close to the object, the fluid model to be employed in the following is not applicable. A much more elaborate description is then necessary which is beyond the scope of the present investigation.

\section{FORMULATION}

Consider a point charge $q$ moving through a stationary plasma with velocity $-v \mathbf{e}_{x}$. With respect to the coordinate system fixed to the point charge, the steady perturbed electron motion is governed by the following equations:

$$
\begin{gathered}
n_{0} \nabla \cdot \mathbf{u}_{\mathrm{e}}+v\left(\partial n_{\mathrm{e}} / \partial x\right)=0, \\
m_{\mathrm{e}} n_{0} v\left(\partial \mathbf{u}_{\mathrm{e}} / \partial x\right)=-\nabla p_{\mathrm{e}}-e n_{0} \mathbf{E}-\nu \mathbf{u}_{\mathrm{e}}, \\
\nabla p_{\mathrm{e}}=m_{\mathrm{e}} a_{0}^{2} \nabla n_{\mathrm{e}} \\
\nabla \cdot \mathbf{E}=\left(-e n_{\mathrm{e}} / \epsilon_{0}\right)+\left[q \delta(\mathbf{x}) / \epsilon_{0}\right], \\
\nabla \times \mathbf{E}=\mathbf{0},
\end{gathered}
$$

where $n_{0}$ is the electron number density at equilibrium, $m_{\mathrm{e}}$ is the electron mass, $a_{0}$ is the equilibrium sound speed, and $n_{\mathrm{e}}, \mathbf{u}_{\mathrm{e}}, p_{\mathrm{e}}$ are the perturbed electron number density, velocity, and pressure, respectively.

\section{ELECTRON SCREENING}

The screening of the point charge $q$ by the electrons will first be considered. By taking divergence of Eq. (2) and using Eqs. (1), (3), and (4), the fol- 
lowing equation for the electron number density can be obtained:

$$
\begin{array}{r}
\left(1-M^{2}\right) \frac{\partial^{2} n_{\mathrm{e}}}{\partial x^{2}}+\frac{\partial^{2} n_{\mathrm{e}}}{\partial y^{2}}+\frac{\partial^{2} n_{\mathrm{e}}}{\partial z^{2}}-\frac{\nu v}{n_{0} m_{\mathrm{e}} a_{0}^{2}} \frac{\partial n_{\mathrm{e}}}{\partial x} \\
-\frac{e^{2} n_{\mathrm{o}}}{\epsilon_{0} m_{\mathrm{e}} a_{0}^{2}} n_{\mathrm{e}}=\frac{-e n_{\mathrm{n}} q}{\epsilon_{0} m_{\mathrm{e}} a_{0}^{2}} \delta(\mathbf{x}),
\end{array}
$$

where the Mach number $M=v / a_{0}$.

The solution of Eq. (6) can be divided into three cases according to (1) $M^{2}<1$, subsonic; (2) $M^{2}=1$, transonic; (3) $M^{2}>1$, supersonic.

\section{(1) Subsonic Case $(M<1)$}

Let us make the following change of variables:

$$
\begin{gathered}
\zeta=\frac{x}{\left(1-M^{2}\right)^{\frac{1}{2}}}, \quad \alpha=\frac{\nu v}{n_{0} m_{\mathrm{e}} a_{0}^{2}\left(1-M^{2}\right)^{\frac{1}{2}}}, \\
\beta^{2}=\frac{e^{2} n_{0}}{\epsilon_{0} m_{\mathrm{e}} a_{0}^{2}}, \quad n_{\mathrm{e}}=e^{\frac{1}{\alpha} \alpha \zeta} \psi .
\end{gathered}
$$

Then Eq. (6) becomes

$$
\begin{aligned}
\frac{\partial^{2} \psi}{\partial \zeta^{2}}+\frac{\partial^{2} \psi}{\partial y^{2}}+ & \frac{\partial^{2} \psi}{\partial z^{2}}-\left(\frac{\alpha^{2}}{4}+\beta^{2}\right) \psi \\
& =\frac{-e n_{0} q}{\epsilon_{0} m_{e} a_{0}^{2}\left(1-M^{2}\right)^{\frac{1}{2}}} \delta(\zeta) \delta(y) \delta(z)
\end{aligned}
$$

The solution of Eq. (7) is well known;

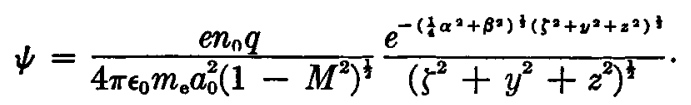

Therefore,

$$
n_{\mathrm{e}}=\frac{e n_{0} q}{4 \pi \epsilon_{0} m_{\mathrm{e}} a_{0}^{2}\left(1-M^{2}\right)^{\frac{1}{2}}} \frac{\exp \left\{\frac{\alpha x}{\left.2\left(1-M^{2}\right)^{\frac{1}{2}}-\left(\frac{1}{4} \alpha^{2}+\beta^{2}\right)^{\frac{1}{3}}\left[\frac{x^{2}}{1-M^{2}}+y^{2}+z^{2}\right]^{\frac{1}{1}}\right\}}\right.}{\left[\frac{x^{2}}{\left(1-M^{2}\right)}+y^{2}+z^{2}\right]^{\frac{1}{2}}} .
$$

Let us examine the nature of solution as given by Eq. (9). First, let us neglect the collision of ions and electrons, i.e., $\nu \rightarrow 0$, then Eq. (9) becomes

$$
\begin{aligned}
n_{\bullet}= & \frac{e n_{0} q}{4 \pi \epsilon_{0} m_{\mathrm{e}} a_{0}^{2}\left(1-M^{2}\right)^{\frac{1}{2}}} \\
& \frac{\exp \left\{-\beta\left[\frac{x^{2}}{\left(1-M^{2}\right)^{\frac{1}{2}}}+y^{2}+z^{2}\right]^{3}\right\}}{\left[\frac{x^{2}}{\left(1-M^{2}\right)}+y^{2}+z^{2}\right]^{t}} .
\end{aligned}
$$

Equation (10) shows that within the linearized theory $n_{\mathrm{o}}$ is symmetric with respect to the $y-z$ plane. Thus, it follows that there is no resultant electric field acting on the charge $q$. Since electric force is the only force acting on $q$, the charge experiences no drag.

The above result leads to the conclusion that for a subsonic particle the drag on it is associated with $\nu$. The same conclusion, however, does not hold for supersonic particles, in which case the nature of the electron screening is completely different. For $\nu \neq 0, \mathrm{Eq}$. (9) shows a "wake-like" structure in the disturbance. This wake is responsible for the drag on the moving charged particle.

\section{(2) Transonic Case $(M=1)$}

In this case, Eq. (6) becomes

$\frac{\partial^{2} n_{\mathrm{o}}}{\partial y^{2}}+\frac{\partial^{2} n_{\mathrm{e}}}{\partial z^{2}}-\gamma \frac{\partial n_{\mathrm{e}}}{\partial x}-\beta^{2} n_{\mathrm{e}}=-\frac{e n_{0} q}{\epsilon_{0} m_{\mathrm{e}} a_{0}^{2}} \delta(\mathrm{x})$,

where $\gamma=\nu v /\left(n_{0} m_{\mathrm{e}} a_{0}^{2}\right)$.
Let $n_{\mathrm{o}}=\chi \exp \left(-\beta^{2} x / \gamma\right)$, then $\chi$ satisfies

$$
\begin{aligned}
\left(\partial^{2} \chi / \partial y^{2}\right)+\left(\partial^{2} \chi / \partial z^{2}\right) & -\gamma(\partial \chi / \partial x) \\
& =\left(-e n_{0} q / \epsilon_{0} m_{\ominus} a_{0}^{2}\right) \delta(x) .
\end{aligned}
$$

The solution of Eq. (12) is well known;

$$
\begin{aligned}
& \chi=\left(e n_{0} q / 4 \pi \epsilon_{0} m_{\mathrm{e}} a_{0}^{2} x\right) \\
& \cdot \exp \left[-\gamma\left(y^{2}+z^{2}\right) / 4 x\right] H(x),
\end{aligned}
$$

where $H(x)$ is the unit step function. Hence,

$$
\begin{aligned}
n_{\mathrm{e}}= & \left(e n_{0} q / 4 \pi \epsilon_{0} m_{\mathrm{e}} a_{0}^{2} x\right) \\
& \cdot \exp \left[-\gamma\left(y^{2}+z^{2}\right) / 4 x-\beta^{2} x / \gamma\right] H(x) .
\end{aligned}
$$

Equation (14) indicates that the charged particle has no influence on the plasma charge density over the half space $x<0$. Later, it will be shown that this corresponds to the transition between subsonic and supersonic case.

\section{(3) Supersonic Case $(M>1)$}

In this case, after making the following change of variables, Eq. (6) can be written as

$$
\begin{aligned}
\zeta= & \frac{x}{\left(M^{2}-1\right)^{\frac{1}{2}}}, \quad \lambda=\frac{w}{n_{0} m_{\mathrm{\theta}} a_{0}^{2}\left(M^{2}-1\right)^{\frac{1}{2}}}, \\
n_{\bullet}=\Phi & \exp \left(-\frac{1}{2} \lambda \zeta\right) \\
& -\frac{\partial^{2} \Phi}{\partial \zeta^{2}}+\frac{\partial^{2} \Phi}{\partial y^{2}}+\frac{\partial^{2} \Phi}{\partial z^{2}}-\left(\beta^{2}-\frac{\lambda^{2}}{4}\right) \Phi \\
& =-\frac{e n_{0} q}{\epsilon_{0} m_{\mathrm{e}} a_{0}^{2}\left(M^{2}-1\right)^{\frac{1}{2}}} \delta(\zeta) \delta(y) \delta(z) .
\end{aligned}
$$


The solution of Eq. (15) depends on the relative magnitude of $\beta^{2}$ and $\frac{1}{4} \lambda^{2}$.

(a) $\beta^{2}>\frac{1}{4} \lambda^{2}$. Take Fourier transform of Eq. (15) in $y$ and $z$. Then

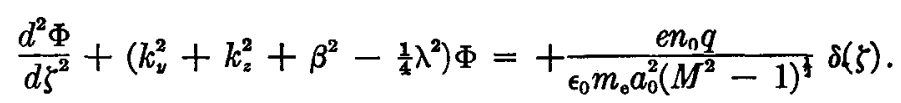

The boundary conditions on $\Phi$ are

$$
\begin{gathered}
\Phi \rightarrow 0, \quad \zeta \rightarrow-\infty, \\
\Phi \text { is bounded, } \zeta \rightarrow+\infty, \\
{\left[\frac{d \Phi}{d \zeta}\right]_{\text {jump }}=\frac{e n_{0} q}{\epsilon_{0} m_{\mathrm{e}} a_{0}^{2}\left(M^{2}-1\right)^{\frac{2}{2}}} \text { at } \zeta=0 .}
\end{gathered}
$$

The unique solution which satisfies Eq. (16), and the above conditions is

$$
\Phi= \begin{cases}0 & \zeta<0 \\ \frac{e n_{0} q}{\epsilon_{0} m_{\mathrm{e}} a_{0}^{2}\left(M^{2}-1\right)^{\frac{1}{2}} \frac{1}{\left(k_{y}^{2}+k_{z}^{2}+\beta^{2}-\frac{1}{4} \lambda^{2}\right)^{\frac{1}{2}}} \sin \left(k_{\nu}^{2}+k_{z}^{2}+\beta^{2}-\frac{1}{4} \lambda^{2}\right)^{\frac{1}{\zeta}} \zeta} & \zeta>0 .\end{cases}
$$

Inverting the Fourier transform, we have

$$
\Phi=\left\{\begin{array}{l}
\frac{e n_{0} q}{2 \pi \epsilon_{0} m_{\mathrm{e}} a_{0}^{2}\left(M^{2}-1\right)^{\frac{1}{3}}} \frac{\cos \left[\left(\beta^{2}-\frac{1}{4} \lambda^{2}\right)^{\frac{1}{2}}\left(\zeta^{2}-y^{2}-z^{2}\right)^{\frac{1}{3}}\right]}{\left(\zeta^{2}-y^{2}-z^{2}\right)^{\frac{1}{2}}} \zeta^{2}>y^{2}+z^{2}, \quad \zeta>0 . \\
0 \quad \text { elsewhere }
\end{array}\right.
$$

Therefore,

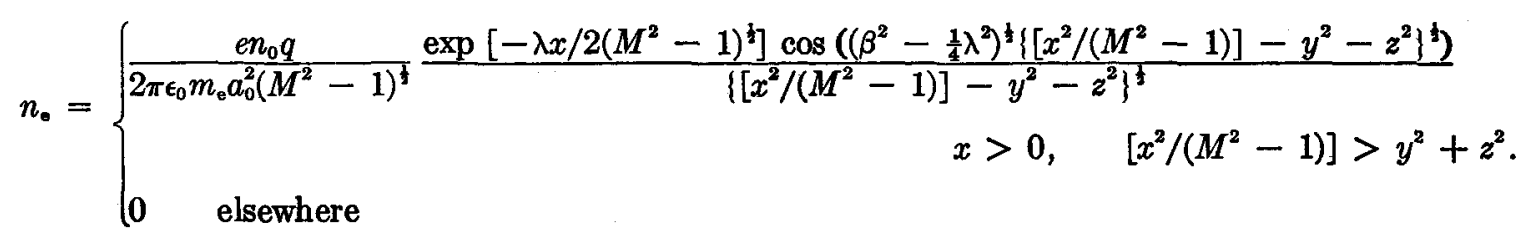

(b) $\beta^{2}<\frac{1}{4} \lambda^{2}$. For this case, by the same procedure as above, we have

$$
n_{\mathrm{e}}=\left\{\begin{array}{c}
\frac{e n_{0} q}{2 \pi \epsilon_{0} m_{\mathrm{e}} a_{0}^{2}\left(M^{2}-1\right)^{\frac{1}{2}}} \frac{\exp \left[\frac{-\lambda x}{\left.2\left(M^{2}-1\right)^{\frac{1}{2}}\right]} \cosh \left\{\left(\frac{1}{4} \lambda^{2}-\beta^{2}\right)^{\frac{1}{2}}\left[\frac{x^{2}}{\left(M^{2}-1\right)}-y^{2}-z^{2}\right]^{\frac{1}{2}}\right\}\right.}{\left\{\left[x^{2} /\left(M^{2}-1\right)\right]-y^{2}-z^{2}\right\}^{\frac{1}{3}}} \\
x>0, \quad x^{2}>\left(M^{2}-1\right)\left(y^{2}+z^{2}\right) . \\
\quad \text { elsewhere }
\end{array}\right.
$$

The solutions given by Eqs. (19) and (20) show that the disturbance on the electron charge density generated by the charged particle is confined to the cone $x^{2}>\left(M^{2}-1\right)\left(y^{2}+z^{2}\right)$. For $\frac{1}{4} \lambda^{2}<\beta^{2}$, the disturbance oscillates before being completely damped out; while for $\frac{1}{4} \lambda^{2}>\beta^{2}$ the equation yields a completely damped solution. The surface of the cone $x>0, x^{2}>\left(M^{2}-1\right)\left(y^{2}+z^{2}\right)$ is a singular surface where the linearized solution fails to hold.

\section{CONTINUITY OF SOLUTION}

In the above treatment, three solutions are found according to $M<1, M=1$, and $M>1$. However, it is simple and straightforward to show that

$$
\begin{aligned}
\underset{M \rightarrow 1}{L t} \frac{e n_{0} q}{4 \pi \epsilon_{0} m_{\mathrm{o}} a_{0}^{2}\left(M^{2}-1\right)} \frac{\exp \left(\alpha x / 2\left(1-M^{2}\right)^{\frac{1}{2}}-\left[\alpha^{2} /\left(4-\beta^{2}\right)\right]^{\frac{1}{2}}\left\{\left[x^{2} /\left(M^{2}-1\right)\right]+y^{2}+z^{2}\right\}^{\frac{3}{3}}\right)}{\left[x^{2} /\left(1-M^{2}\right)+y^{2}+z^{2}\right]^{\frac{1}{2}}} \\
\rightarrow \frac{e n_{0} q}{4 \pi \epsilon_{0} m_{\mathrm{e}} a_{0}^{2} x} \exp \left\{\left[-\gamma\left(y^{2}+z^{2}\right) / 4 x\right]-\beta^{2} x / \gamma\right\} H(x),
\end{aligned}
$$




$$
\begin{aligned}
\underset{M f \rightarrow 1}{L t} \frac{e n_{0} q}{2 \pi \epsilon_{0} m_{\mathrm{e}} a_{0}^{2}\left(M^{2}-1\right)^{\frac{1}{2}}} \frac{\exp \left[-\lambda x / 2\left(M^{2}-1\right)^{\frac{1}{2}}\right] \cosh \left(\left[\lambda^{2} /\left(4-\beta^{2}\right)\right]^{\frac{1}{2}}\left\{\left[x^{2} /\left(M^{2}-1\right)\right]-y^{2}-z^{2}\right\}^{\frac{1}{2}}\right)}{\left[x^{2} /\left(M^{2}-1\right)-y^{2}-z^{2}\right]^{\frac{1}{2}}} \\
\rightarrow \frac{e n_{0} q}{4 \pi \epsilon_{0} m_{\mathrm{e}} a_{0}^{2} x} \exp \left\{\left[-\gamma\left(y^{2}+z^{2}\right) / 4 x\right]-\beta^{2} x / \gamma\right\} H(x)
\end{aligned}
$$

Hence, the subsonic, transonic, and supersonic solutions, Eqs. (9), (14), (18), and (19), continue one into the other as Mach number $M$ varies from smaller to greater than unity. There is no breakdown in the mathematical solution for all values of $v$, even though the nature of the disturbances created in the subsonic and supersonic cases differs markedly.

\section{VELOCITY FIELD IN SUPERSONIC CASE}

Now let us determine the perturbed velocity field in the supersonic case. By taking the curl of Eq. (2), we have

$$
\operatorname{curl} \mathbf{u}_{\mathrm{e}}=\mathbf{0} .
$$

Hence, let $\mathbf{u}_{\mathrm{e}}=\nabla \Psi$ and from Eq. (1) $\Psi$ satisfies

$$
\nabla^{2} \Psi=-\left(v / n_{0}\right)\left(\partial n_{\mathrm{e}} / \partial x\right)
$$

Take Fourier transform of Eq. (21) in $y$ and $z$, and using Eq. (17), then

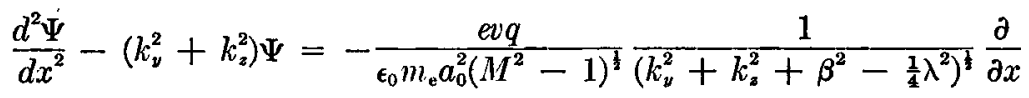

$$
\begin{aligned}
& \cdot\left\{\exp \left[\frac{-\lambda x}{2\left(M^{2}-1\right)^{\frac{1}{2}}}\right] \sin \frac{\left(k_{y}^{2}+k_{z}^{2}+\beta^{2}-\frac{1}{4} \lambda^{2}\right)^{\frac{1}{2}} x}{\left(M^{2}-1\right)^{\frac{1}{2}}}\right\}, \quad x>0 \\
& =0, \quad x<0 \text {. }
\end{aligned}
$$

The boundary conditions of $\Psi$ are

$$
\Psi \rightarrow 0, \quad x \rightarrow-\infty, \quad \Psi \text { is bounded as } x \rightarrow \infty, \quad \Psi \text { and } d \Psi / d x \text { are continuous. }
$$

It is straightforward to obtain the solution of Eq. (22) satisfying the above boundary conditions. The full solution is quite lengthy and will not be written out here. However, for $\nu=0$, on inverting the transform,

$$
\Psi= \begin{cases}\frac{e v q}{4 \pi \epsilon_{0} m_{\mathrm{e}} a_{0}^{2}} \int_{0}^{\infty} \frac{\exp (k x) J_{0}(k \rho) k d k}{\left(\beta^{2}+k^{2} M^{2}\right)} ; \quad \rho^{2}=y^{2}+z^{2} ; \quad x<0, \\ -\frac{e v q}{4 \pi \epsilon_{0} m_{\mathrm{e}} a_{0}^{2}} \int_{0}^{\infty} \frac{\exp (-k x)+2 \cos \left[\left(k^{2}+\beta^{2}\right)^{\frac{1}{2}} x /\left(M^{2}-1\right)\right]}{\left(\beta^{2}+k^{2} M^{2}\right)} J_{0}(k \rho) k d k ; \quad x>0 .\end{cases}
$$

From Eq. (23) for $x<0$, it is clear that $\nabla \cdot u_{e}=0$. Thus, in front of the Mach cone, the plasma behaves as an incompressible fluid. The motion of the electron fluid is determined by a balance of inertial and electrostatic forces.

\section{ELECTROHYDRODYNAMIC DRAG}

To determine the drag on the charged particle $q$, it is necessary to find the electric field at the origin due to $n_{\mathrm{e}}$. Let $\mathbf{E}=-\nabla \varphi$; then

$$
\nabla^{2} \varphi=\left(e n_{\mathrm{e}}\right) / \epsilon_{0}
$$

where $n_{\mathrm{e}}$ is given by Eq. (9) or (19), according to whether $M$ is less than or greater than unity.

\section{(1) Subsonic Case}

Take Fourier transform of Eq. (24) in $y$ and $z$; then

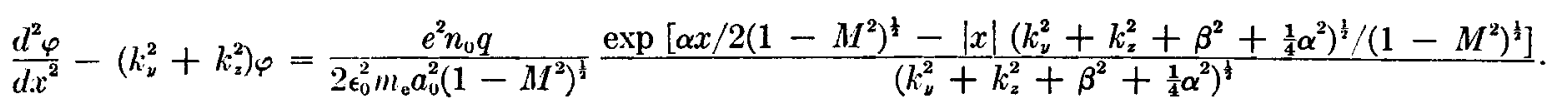


The boundary conditions on $\varphi$ are

$$
\varphi \rightarrow 0, \quad x \rightarrow \pm \infty, \quad \varphi \text { and } d \varphi / d x \text { are continuous. }
$$

Solution of Eq. (25) satisfying the above boundary conditions can be obtained by an elementary technique in ordinary differential equation. From this solution, it is straightforward to get

$\left[\frac{\partial \varphi}{\partial x}\right]_{x=0}=\frac{e^{2} n_{0} q\left\{\alpha\left(1-M^{2}\right)^{\frac{1}{2}}\left(k_{y}^{2}+k_{z}^{2} \frac{1}{2}\left(k_{y}^{2}+k_{z}^{2}+\beta^{2}+\frac{1}{4} \alpha^{2}\right)^{\frac{1}{2}}-\frac{1}{2} \alpha \beta^{2}+\alpha\left(k_{y}^{2}+k_{z}^{2}\right)\left[M^{2} /(2-1)\right]\right\}\right.}{2 \epsilon_{0}^{2} m_{\mathrm{e}} a_{0}^{2}\left(k_{y}^{2}+k_{z}^{2}+\beta^{2}+\frac{1}{4} \alpha^{2}\right)\left\{\left[\beta^{2}+\frac{1}{2} \alpha^{2}+\left(k_{y}^{2}+k_{z}^{2}\right) M^{2}\right]^{2}-\alpha^{2}\left(k_{y}^{2}+k_{z}^{2}+\beta^{2}+\frac{1}{4} \alpha^{2}\right)\right\}}$

On inverting the Fourier transform of Eq. (26),

where

$$
E_{x}(0)=\frac{e^{2} n_{0} q \alpha}{4 \pi \epsilon_{0}^{2} m_{\bullet} a_{0}^{2}} Z\left(\alpha, \beta, M^{2}\right)
$$

$$
Z\left(\alpha, \beta, M^{2}\right)=\int_{0}^{\infty} \frac{\frac{1}{2} \beta^{2}+\left(1-\frac{1}{2} M^{2}\right) x-\left(1-M^{2}\right)^{\frac{1}{2}} x^{\frac{1}{2}}\left(x+\beta^{2}+\frac{1}{4} \alpha^{2}\right)^{\frac{1}{2}}}{2\left(x+\beta^{2}+\frac{1}{4} \alpha^{2}\right)\left\{\left[\beta^{2}+\alpha^{2} /\left(2+x M^{2}\right)\right]^{2}-\alpha^{2}\left(x+\beta^{2}+\frac{1}{4} \alpha^{2}\right)\right\}} d x
$$

Therefore, the drag on the moving charge is equal to

$$
q E_{x}(0)=\frac{e^{2} n_{0} q^{2} \alpha}{4 \pi \epsilon_{0}^{2} m_{\mathrm{e}} a_{0}^{2}} Z\left(\alpha, \beta, M^{2}\right)
$$

From Eq. (29), it is clear that if $\nu \rightarrow 0$ there is no drag on the particle, which confirms our earlier argument.

\section{(2) Supersonic Case}

In this case, instead of Eq. (27), we have

$\frac{d^{2} \varphi}{d x^{2}}-\left(k_{y}^{2}+k_{z}^{2}\right) \varphi=\left\{\begin{array}{l}\frac{e^{2} q n_{0}}{\epsilon_{0}^{2} m_{\mathrm{e}} a_{0}^{2}\left(M^{2}-1\right)^{\frac{1}{2}}} \frac{\exp \left[-\lambda x / 2\left(M^{2}-1\right)^{\frac{1}{2}}\right]}{\left(k_{y}^{2}+k_{z}^{2}+\beta^{2}-\frac{1}{4} \lambda^{2}\right)^{\frac{1}{1}}} \sin \left[\frac{\left(k_{\nu}^{2}+k_{z}^{2}+\beta^{2}-\frac{1}{4} \lambda^{2}\right)^{\frac{1}{2}} x}{\left(M^{2}-1\right)^{\frac{1}{2}}}\right] x>0 \\ 0 \quad x<0 .\end{array}\right.$

The boundary conditions on $\varphi$ are

$$
\varphi \rightarrow 0, \quad x \rightarrow-\infty, \quad \varphi \text { is bounded as } x \rightarrow+\infty, \quad \varphi \text { and } d \varphi / d x \text { are continuous. }
$$

The solution of Eq. (30) which satisfies the boundary conditions is $x \geq 0$,

$$
\begin{aligned}
& \varphi=-\frac{e^{2} q n_{0}\left[\lambda\left(M^{2}-1\right)^{\frac{1}{2}}\left(k_{y}^{2}+k_{z}^{2}\right)^{\frac{1}{2}}+\left(k_{y}^{2}+k_{z}^{2}\right) M^{2}+\beta^{2}\right] \exp \left[-\left(k_{y}^{2}+k_{z}^{2}\right)^{\frac{1}{2}} x\right]}{2 \epsilon_{0} m_{\mathrm{e}} a_{0}^{2}\left(k_{y}^{2}+k_{z}^{2}\right)^{\frac{1}{2}}\left[\left\{\frac{1}{2} \lambda^{2}-\left(k_{y}^{2}+k_{z}^{2}\right) M^{2}-\beta^{2}\right\}^{2}+\lambda^{2}\left(k_{y}^{2}+k_{z}^{2}+\beta^{2}-\frac{1}{4} \lambda^{2}\right)\right]} \\
& +\frac{e^{2} q n_{0}\left(M^{2}-1\right)^{\frac{1}{2}} \exp \left[-\lambda x / 2\left(M^{2}-1\right)^{\frac{1}{2}}\right]}{\epsilon_{0}^{2} m_{\mathrm{e}} a_{0}^{2}\left(k_{y}^{2}+k_{z}^{2}+\beta^{2}-\frac{1}{4} \lambda^{2}\right)^{\frac{1}{2}}\left[\left\{\frac{1}{2} \lambda^{2}-\left(k_{y}^{2}+k_{z}^{2}\right) M^{2}-\beta^{2}\right\}^{2}+\lambda^{2}\left(k_{y}^{2}+k_{z}^{2}+\beta^{2}-\frac{1}{4} \lambda^{2}\right)\right]} \\
& \cdot\left\{\left[\frac{1}{2} \lambda^{2}-\left(k_{y}^{2}+k_{z}^{2}\right) M^{2}-\beta^{2}\right] \sin \frac{\left(k_{y}^{2}+k_{z}^{2}+\beta^{2}-\frac{1}{4} \lambda^{2}\right)^{\frac{1}{2}} x}{\left(M^{2}-1\right)^{\frac{1}{2}}}\right. \\
& \left.+\lambda\left(k_{\nu}^{2}+k_{z}^{2}+\beta^{2}-\frac{1}{4} \lambda^{2}\right)^{\frac{1}{2}} \cos \frac{\left(k_{y}^{2}+k_{z}^{2}+\beta^{2}-\frac{1}{4} \lambda^{2}\right)^{\frac{1}{2}} x}{\left(M^{2}-1\right)^{\frac{1}{2}}}\right\} \\
& x \leq 0, \quad \varphi=\frac{e^{2} q n_{0}\left[\lambda\left(M^{2}-1\right)^{\frac{1}{2}}\left(k_{\nu}^{2}+k_{z}^{2}\right)^{\frac{1}{2}}-\left(k_{\nu}^{2}+k_{z}^{2}\right) M^{2}-\beta^{2}\right] \exp \left[\left(k_{y}^{2}+k_{z}^{2}\right)^{\frac{1}{2}} x\right]}{2 \epsilon_{0}^{2} m_{\mathrm{e}} a_{0}^{2}\left(k_{y}^{2}+k_{z}^{2}\right)^{\frac{1}{2}}\left[\left(\left\{\frac{1}{2} \lambda^{2}-\left(k_{y}^{2}+k_{z}^{2}\right) M^{2}-\beta^{2}\right\}^{2}+\lambda^{2}\left(k_{u}^{2}+k_{z}^{2}+\beta^{2}-\frac{1}{4} \lambda^{2}\right)\right]\right.} .
\end{aligned}
$$

From Eq. (31), for $\nu=0$, it is straightforward to obtain

Therefore,

$$
E_{x}(0)=\frac{e^{2} q n_{0}}{8 \pi \epsilon_{0}^{2} m_{\mathrm{e}} a_{0}^{2} M^{2}} \ln \left[k^{2} M^{2}+\beta^{2}\right]_{k=0}^{k=\infty} .
$$

$$
\operatorname{Drag}=\frac{e^{2} q^{2} n_{0}}{8 \pi \epsilon_{0}^{2} m_{\mathrm{e}} a_{0}^{2} M^{2}} \ln \left[\frac{k_{\max }^{2} M^{2}+\beta^{2}}{\beta^{2}}\right]
$$

where the upper limit has been replaced by $k_{\max }$. 


\section{SUMMARY}

Within the framework of a linearized continuum description, the disturbance produced by a moving charged particle on a plasma has been studied. The phenomena involved can be divided into subsonic, transonic, and supersonic regimes. For a subsonic particle, the screening electrons form a wake-like structure which is responsible for the drag on the particle. Electron screening around a supersonic particle is confined to the downstream Mach cone. In front of the Mach cone, the electrons behave as an incompressible fluid. Their motion is deter- mined by a balance of inertial and electrostatic forces. The drag force on the particle in this case has a logarithmic divergence. It was also shown that the fluid model used was capable of describing any particle velocity without serious mathematical breakdown, even when Mach number approaches unity.

\section{ACKNOWLEDGMENTS}

The author is grateful to Professor W. D. Rannie for his encouragement and to Professor R. Gould for his valuable comments in the course of this work. 